\title{
Doing Gender im IT-Design - Zur Problematik der (Re-)Konstruktion von Differenz
}

\author{
Saskia Sell \\ Institut für Publizistik- und Kommunikationswissenschaft, Freie Universität Berlin
}

\section{$1 \quad$ Das Konzept des Doing Gender aus der Perspektive der Gender Media Studies}

Eine Auseinandersetzung mit der Genderperspektive im Bereich HCI, Web Usability und User Experience birgt immer auch die Gefahr, missverstanden zu werden und dadurch Geschlechterunterschiede bzw die Geschlechterdichotomie ungewollt zu reifizieren. Durch eine Überbetonung von Differenz zwischen zwei binär gedachten Geschlechtern kann das Bild entstehen, dass diese Unterschiede Voraussetzung für eine erfolgreiche Gestaltung von $\mathrm{Be}$ dienoberflächen und Online-Kommunikation seien. Daher ist es wichtig, kritische Perspektiven aus den Gender Studies stets im Blick zu halten, um die eigene Position kritisch zu hinterfragen und nicht ungewollt herrschende Geschlechterkonstruktionen zu reproduzieren.

Bedürfnisse, Interessen und Kontexte von Männern und Frauen werden häufig explizit unterschiedlich gedacht. In klassischen Denk- und Deutungsmustern des Differenzfeminismus in der Tradition Kristevas oder Irigarays und theoretischer Strömungen der Geschlechterforschung, die im Kern ein essentialistisches Verständnis von Geschlecht zugrunde legen, wird eine binäre Differenz zwischen Mann und Frau, ,wenn nicht qua Biologie, so doch qua historisch-gesellschaftlicher Sozialisation" (Lünenborg und Maier, 2013, S. 19), festgeschrieben. Diese Interpretation greift zu kurz und wird den aufgerufenen Auseinandersetzungen und kreativen Spielarten mit dem sozialen Geschlecht nicht gerecht.

Feministische Strömungen, die sich auf das hier angesprochene Differenzmodell berufen, üben häufig Kritik an zentralen Forderungen nach Angleichung weiblichen Handelns an in rein männlichen Zusammenhängen konstruierte Normen, Handlungsweisen und Strukturen. Nicht die Annäherung an das Modell des Männlichen, das als bereits gesetzte und naturalisierte Norm unhinterfragt bleibt, soll das Ziel feministischer Politik sein, sondern die Anerkennung des Weiblichen als dezidiert Anderem.

Diese gleichermaßen einseitige Betrachtungsweise ist nicht minder problematisch, weshalb im Folgenden knapp weitere zentrale Theorieansätze der Gender Media Studies dargestellt werden, um hinzuführen zum Konzept des Doing Gender, das in der Auseinandersetzung mit der Gestaltung von Kommunikationstechnologie den Spielraum, der jede Art von Design erst ermöglicht, erweitert und deterministischen Ansätzen eine emanzipatorische Perspektive gegenüberstellt. Zwischen sozialtheoretischen Konzeptionen und anwendungsorientierten 
Fragestellungen findet somit, im Hinblick auf die Konstruktion von Geschlecht, eine Verknüpfung statt.

Die Problematik der (Re-)Konstruktion von Differenz durch Kommunikationsdesign soll entlang dieser zentralen Theorieansätze der Geschlechterforschung kritisch diskutiert werden.

\subsection{Vom Gleichheitsansatz zum Differenzansatz}

Der einleitend angesprochene Differenzansatz hat sich innerhalb der Geschlechterforschung als Gegenpol zum ursprünglichen Gleichheitsansatz entwickelt. Letzterer bildet den erkenntnistheoretischen Ursprung der kommunikationswissenschaftlichen Geschlechterforschung (damals noch „Frauenforschung“) und verfolgte das Anliegen, „Diskriminierungen von Frauen in den Medien in Form von Unterrepräsentanz einerseits und Stereotypisierung und Trivialisierung andererseits aufzuzeigen und mehr Gleichheit in Bezug auf die Darstellung der Geschlechter zu erreichen“ (Röser und Müller, 2012, S. 38, unter Rückgriff auf Klaus, 2001). Neben der Untersuchung von Formen der Repräsentation - oder NichtRepräsentation, wie Tuchman in ihrem bereits 1978 erschienenen Aufsatz „The Symbolic Annihilation of Women by the Mass Media" treffend dargestellt hat - von Frauen in den Medien wurde der Gleichheitsansatz vor allem in den 1980er Jahren im Rahmen der Berufsfeldforschung, die Geschlechterstrukturen - beispielsweise Ungleichheiten in der Bezahlung, geringere Anzahl von Frauen in Macht- und Entscheidungspositionen und damit geringere Handlungsspielräume etc. - im Medienproduktionskontext untersuchte, zur Grundlage empirischer Studien (u. a. Becher et al., 1981; Neverla, 1983; Neverla und Kanzleiter, 1984).

Von den Annahmen des Gleichheitsfeminismus unterscheiden sich differenzfeministische Strömungen in Sozialtheorie und Medienpraxis. Ungleichheit wird „nicht (nur) unfreiwillig erfahren, sondern (auch) bewusst und aktiv hergestellt" (Lünenborg und Maier, 2013, S. 28). Etwa durch Fragen nach dezidiert „,weiblicher“ Mediennutzung und damit verbundener differenter Aneignung von IT wurde unter Betonung des Unterschieds der Geschlechter nach Formen des „weiblichen Schreibens“, des „weiblichen Journalismus“ oder „weiblicher“ PRKommunikation, die gleichgesetzt wurde mit mehr „Freundlichkeit“, gefragt. Neben kritischen Studien, die derartige als typisch weiblich konstruierte Verhaltensweisen und Formen der ,geschlechtsgebundenen Besonderung“ (Lünenborg und Maier 2013, S. 29) im Zusammenhang mit Karrierehemmnissen sahen, gab es eine Reihe positivistischer Ansätze, die in der Interpretation von Ergebnissen aus Mediennutzungsstudien die vorab angenommene Differenz durch unterschiedliche Nutzungsweisen von Medienprodukten bestätigt sahen. Aktuellere Perspektiven der kommunikationswissenschaftlichen Geschlechterforschung betonen jedoch, dass sich weder das Rezeptions- oder Mediennutzungsverhalten noch bestimmte Darstellungsformen oder Genrestrukturen angemessen entlang einer als dualistisch verstandenen Geschlechterordnung verstehen lassen (ebd., S. 30). Es bedarf an dieser Stelle also eines Perspektivenwechsels.

\subsection{Dekonstruktion und Doing Gender}

Aus der Auseinandersetzung zwischen Gleichheits- und Differenzdeutungen heraus entwickelten sich, besonders in Abgrenzung zum Differenzfeminismus, erweiterte emanzipatorische Ansätze entlang der Konzepte der Diskurstheorie und des interaktionistischen Konstruk- 
tivismus (Lünenborg und Maier, 2013, S. 78 ff.; Gildemeister, 2008, S. 172 ff.). Geschlecht gilt hier, der Theoriearbeit Butlers folgend, nicht als etwas, das biologisch und/oder qua Sozialisation festgelegt ist, sondern als performativer Akt. Gender ist also nicht Essenz, sondern „Identität, die durch eine stilisierte Wiederholung von Akten zustande kommt“ (Butler, 2002, S. 302). Erst durch Performativität, durch die Wiederholung bestimmter Handlungsmuster in Verbindung mit der Akzeptanz der Deutungshoheit diskursiver Strömungen, die diese Handlungsmuster als „männlich“ oder ,weiblich“ konnotieren, wird Geschlecht und werden mit Geschlecht verbundene gesellschaftliche Ordnungsstrukturen, interaktiv (re)produziert. Dieses Verständnis der ,sozialen Konstruktion von Geschlecht“ hat sich seit den frühen 1990er Jahren sowohl in der Geschlechterforschung als auch in der Geschlechterpolitik stark verbreitet, wobei der Konstruktionsbegriff im Bereich der Sozialtheorie unterschiedlich interpretiert und definiert und oft mit diskurstheoretischen Erklärungsmustern gleichgesetzt wird (Gildemeister, 2008, S. 169-170).

Doing Gender - aktives vergeschlechtlichtes Handeln oder reaktives Sich-entlang-vonvorgegebenen-Geschlechterrollenbildern-Verhalten - verleiht der dualistischen Struktur erst ihre Gültigkeit. Männlichkeit und Weiblichkeit sowie die entsprechenden Diskurse werden durch die Verbindung von Handlung, Deutung und Wertung interaktiv hergestellt: Geschlecht hat man nicht, Geschlecht macht man. Dieses Muster innerhalb geschlechtsgebundener Aushandlungsprozesse haben auch Gildemeister und Wetterer (1992) in ihrer mikrosoziologischen Studie anschaulich verdeutlicht. Sie legen in ihrer Analyse den Fokus auf geschlechtsspezifische Sozialisation und Arbeitsteilung mit dem Ziel der Ideologiekritik. Bevor sie Beispiele aus der Berufsfeldforschung anführen, wird die Kategorie Geschlecht hier zunächst sowohl anhand von ethnologischen und kulturanthropologischen Studien als auch auf der Grundlage von biologischen Analysen dekonstruiert. Aus der ersten Perspektive heraus ergibt sich, dass es Kulturen mit drittem Geschlecht gibt, dass Geschlechtswechsel in vielen Kulturen möglich sind und dass die Geschlechtszugehörigkeit in den meisten Fällen aufgrund der Rolle und nicht abhängig von Körpermerkmalen bestimmt wird. Dieser Aufbruch des MannFrau-Dualismus wird gestützt durch biologische Erkenntnisse, die besagen, dass das genetische Geschlecht, das Keimdrüsengeschlecht und das Hormongeschlecht, anders als im Alltagsbewusstsein verankert, nicht übereinstimmen müssen. Für Gildemeister und Wetterer zeigt sich Zweigeschlechtlichkeit daher als soziale Konstruktion, innerhalb derer der Rückgriff auf die Natur lediglich der Legitimationsbeschaffung dient, was auch Scheich in ihrer Analyse bestätigt (Scheich, 1999). Auch sie sehen Doing Gender als aktiv gestaltbaren Handlungszusammenhang und betrachten Geschlecht grundsätzlich als situationsspezifisch, wandelbar und kontextabhängig (Gildemeister und Wetterer, 1992).

Mit diesen neueren Ansätzen verändert sich die Herangehensweise der Geschlechterforschung grundlegend, sie befreit sich aus dem klassischen Dualismus von Männlichkeit und Weiblichkeit und öffnet den Blick für Handlungsweisen und Darstellungsformen, die sich weder dem einen noch dem anderen Schema zuordnen lassen. Zur Dekonstruktion der Annahme, Geschlecht sei etwas Gegebenes, Unveränderliches, Determiniertes, wird vor allem, in Anlehnung an die Tradition der Cultural Studies, innerhalb der Gender Media Studies durch die Wahrnehmung von Brüchen, von kritischer Reflexion oder der Entwicklung ,subversiver Interpretations- und Aneignungsstrategien" beigetragen (Lünenborg und Maier, 2013 und Klaus, 2005).

Um nicht in die Verlegenheit zu kommen, durch wahrgenommene Brüche den Ursprungsdualismus tradierter Narrationen hinterfragen zu müssen, werden allerdings Handlungen und 
Verhaltensweisen, die nicht mit dem klassischen Muster entsprechen, oft diskursiv zunächst als Mängel konstruiert. Wenn sich beispielsweise der Mythos festgeschrieben hat, dass Frauen qua Geschlecht kein Interesse an Technologie haben, führt die gegensätzliche Beobachtung, dass es sehr wohl Frauen mit den entsprechenden Interessen und Fähigkeiten gibt, eher dazu, diese entlang bestehender Deutungsmuster als ,unweiblich“ zu bezeichnen, als dass in letzter Konsequenz die dualistischen Denkweisen und Geschlechterstereotype in ihrer binären Differenz neu überdacht werden. Doch auch diese diskursiv-ordnungspolitische Strategie stößt an ihre Grenzen, was man im Bereich der Gender Media Studies beispielsweise anhand medialer Repräsentationen von Frauen in Machtpositionen ablesen kann - galt Angela Merkel als erste deutsche Bundeskanzlerin zu Beginn ihrer Regierungszeit zunächst als machtvoll, ergo „unweiblich“, fand im Laufe der Jahre eine Veränderung im sie und damit ihre Geschlechtsidentität bezeichnenden Mediensystem statt, wurden Weiblichkeit und Macht nicht mehr als sich wechselseitig ausschließend dargestellt, waren Attribute wie „weiblich“ und „machtvoll“" keine durchgehend sich gegenseitig ausschließenden Zuschreibungen mehr. Dabei wurden auf der Ebene der Repräsentation durchaus Versuche vorgenommen, durch den Fokus auf Weiblichkeit ihre Kompetenz in Frage zu stellen: Gerade die Art der visuellen Medienberichterstattung reproduzierte die Annahme, Weiblichkeit bedinge beschränkte politische Fähigkeiten (Lünenborg et al., 2009, S. 87). Die durch die dualistischen Konzepte entstandenen Deutungsmuster kompetent-unweiblich oder weiblich-inkompetent brechen heute erst langsam auf.

Übertragen auf die Frage nach einer wie auch immer gearteten ,gendersensiblen“ Gestaltung von informationstechnologischen Kommunikationsräumen stellt sich mit Blick auf Genderaspekte also zunächst die Frage nach der eigenen Ausgangsposition und dem professionellen Selbstverständnis. Gilt es im Rahmen einer dezidiert gestalterischen Tätigkeit als Kernaufgabe, auf vermeintlich gesellschaftlich Vorgegebenes $\mathrm{zu}$ reagieren und das eigene Design an als dominant wahrgenommene Deutungsmuster von zweigeschlechtlicher Differenz anzupassen und selbige damit aktiv zu (re-)produzieren, oder werden Formen der binären Vergeschlechtlichung als diskursiv verstärkte performative Akte des Doing Gender verstanden und bewusst neue Impulse gesetzt, die die Konstruiertheit dieser Ordnungsstruktur transparent machen und den dargestellten Dualismus aufbrechen?

Vom Ansatz der dekonstruktivistischen Geschlechteranalyse, die es ermöglicht, derartige Konstruktionen von Differenz transparent zu machen, unterscheiden sich weitere Ansätze aus dem Bereich der IT- und Netzkultur, die sich oft als ,postgender“ geriert. Beispiele lassen sich unter anderem im Selbstverständnis der europäischen Piratenparteien finden, oder in der viel zitierten Hackerethik, die besagt, es käme rein auf Fähigkeiten an und nicht auf sogenannte „Boguskriterien“ wie Geschlecht oder andere soziodemographische Typologisierungen auf Akteursebene. ${ }^{2}$ Der Ansatz erscheint einerseits fair und betont den Wunsch, Menschen nicht qua Geschlecht (oder Klasse, Ethnie, Bildungsgrad etc.) zu bevorzugen oder zu benachteiligen, er ist aber insofern problematisch, als dass er, im Gegensatz zu dekonstruktivistischen Ansätzen, bestehende Differenzkonstruktionen, die sich beispielsweise auch auf den Erwerb von bestimmten Fähigkeiten auswirken können, negiert.

Um sich der Frage zu stellen, ob das Netz oder ob virtuelle Räume neue Möglichkeiten bieten, den klassischen Geschlechterzuschreibungen samt ihrer eingeschriebenen Hierarchie und

\footnotetext{
${ }^{2}$ Vgl. dazu für den deutschsprachigen Raum bspw. die Ausführungen des CCC: http://www.ccc.de/de/hackerethik (letztmaliger Zugriff: 11.05.2014).
} 
bestehender Ungerechtigkeiten $\mathrm{zu}$ entrinnen, ist neben der Anwendung und Erweiterung gestaltungspraktischer Möglichkeiten die Reflexion über die eigene Rolle innerhalb des diskursiven Aushandlungsprozesses notwendig: Was trage ich zur einen oder anderen Entwicklungslinie und zur Relevanzsetzung und Wertung von Geschlecht im Feld der Technologie im Rahmen meiner gestalterischen Tätigkeit bei?

Allen Ansätzen aus der Geschlechterforschung gemein ist ein geteiltes Unbehagen mit der hierarchischen Differenzierung der Gesellschaft entlang von Geschlechteridentitäten. Es geht bei der Geschlechterfrage also immer auch um Forderungen nach Gerechtigkeit und die grundsätzliche Frage nach Hierarchie oder Heterarchie, nach Chancengleichheit und nach selbstbestimmtem Leben innerhalb sozialer Zusammenhänge. Der hier dargestellte Aushandlungsprozess impliziert, dass Technologie im Allgemeinen und Informations- und Kommunikationstechnologie im Besonderen dabei nicht als neutrales Instrument betrachtet werden kann. Im Rahmen der Gestaltung und Aneignung beeinflussen sich technologische Möglichkeiten und kulturelle und/oder politische Dimensionen wechselseitig - was im Folgenden entlang des Co-Emergenzprinzips genauer erörtert wird.

\section{2}

\section{Das Prinzip der Co-Emergenz: wechselseitige Beeinflussung normativer und technologischer Diskursdimensionen}

Winner untersucht in seinen unter dem Titel ,The Whale and the Reactor. A Search for Limits in an Age of High Technology“ 1986 erschienenen zentralen technikphilosophischen Analysen politische und soziale Implikationen von Technologie. Er geht davon aus, dass Entscheidungen, die technologische Entwicklungen betreffen, immer zugleich, ob intendiert oder nicht, politische Entscheidungen sind. Nicht nur das technologisch Machbare, sondern auch normative Aspekte spielen für ihn eine zentrale Rolle in Design- und Konstruktionsprozessen. Besonders anschaulich stellt er dies anhand eines Beispiels aus dem Bereich der Architektur dar: am Bau der von Robert Moses (von den 1920ern bis in die 70er Jahre erfolgreicher Gestalter des öffentlichen Raums in New York) entworfenen Brücken über Zubringerstraßen zu beliebten Naherholungsgebieten auf Long Island. Was hat Brückenbau mit Politik zu tun? Viele der Brücken aus Winners Beispiel wurden für ihre Zeit unüblich niedrig gebaut, so niedrig, dass nur die Autos der (in ihrer Bauzeit vornehmlich weißen) Mittel- und Oberschicht problemlos Zugang zu den weitläufigen Parkanlagen an der Küste Long Islands hatten, während die von der (in ihrer Bauzeit vornehmlich schwarzen) Unterschicht genutzten Busse zu hoch waren, um unter ihnen her fahren zu können. Caro, der Biograph des berühmten Gestalters Moses, erklärt, dass hier bewusst politische Dimensionen in die manifesten Technologien des öffentlichen Raumes eingeschrieben wurden. Moses selbst war über sein Umfeld hinaus bekannt für seine rassistischen und klassenbezogenen Vorurteile, die er, für Winner in diesem Fall besonders deutlich, auch in seine gestalterische Arbeit eingeschrieben hat (Winner, 1986, S. 22-23).

Was für Architektur und Gestaltung öffentlicher Räume gilt, lässt sich übertragen auf den Bereich des Kommunikationsdesigns und die Gestaltung digitaler oder mediatisierter öffentlicher Räume. Nicht nur, was die Offenheit und barrierefreie Zugänglichkeit von digitalen Kommunikationsplattformen und die damit zusammenhängende Frage, wie frei in einem 
bestimmten Kontext kommuniziert werden kann, betrifft, spielen politische Dimensionen auf der Ebene der Gestaltung eine Rolle, sondern auch im Hinblick auf die Frage, ob die konzipierten Nutzungsangebote vergeschlechtlicht werden oder nicht, ob also diejenigen, die die Kommunikationsräume gestalten, sich aktiv an Formen des Doing Gender und an der diskursiven (Re-)Produktion des Mann-Frau-Dualismus beteiligen, oder ob sie diese Differenzkonstruktionen unterlaufen.

Hier wird einmal mehr deutlich, dass kommunikationstechnologische Innovationen, wie beispielsweise das Internet, nicht nur rein technikdeterministisch als Ursache für gesellschaftliche Veränderungen betrachtet werden können. Sie werden in einem Kontinuum sozialer, politischer und kultureller Gemengelagen entworfen, die ihre Gestaltung mindestens genauso stark beeinflussen, wie die Frage danach, was technologisch machbar ist. Dass öffentliche Kommunikationsräume durch die Art ihrer Gestaltung bestimmte Nutzungsformen nahelegen, ist noch kein Garant dafür, dass wir nicht auch andersartige Aneignungsformen, die zum Zeitpunkt der Entwicklung noch nicht mitgedacht wurden, beobachten können. Diese Abgrenzung von einseitig deterministischen Perspektiven greift Wajcman in ihrer technikfeministischen Darstellung auf: „Science and technology embody values, and have the potential to embody different values." (Wajcman, 2004, S. 126).

Dezidiert auf Medieninnovationen bezogen spricht $u$. a. auch Dogruel von Co-Evolution und betont die Wechselwirkung von Gestaltungsprozessen mit sozialen und politischen Strukturen und Diskursen (Dogruel, 2013, S. 328). Der Begriff der Co-Evolution ist allerdings irreführend, tappt man durch die Verwendung der biologischen Evolutionsmetapher allzu leicht wieder in die Determinismusfalle im Hinblick auf „evolutionäre“ Prozesse. Da es sich jedoch um kulturelle Handlungspraktiken auf der Basis individueller Entscheidungsprozesse handelt, erscheint der Emergenzbegriff in diesem Zusammenhang passender. Er befreit einerseits von der Prämisse der Vorhersehbarkeit bestimmter Entwicklungen bzw. von deren kausaler Ableitung aus dem Bestehenden. Andererseits verschleiert er damit auch - im Gegensatz zum Evolutionsbegriff - nicht die Tatsache, dass es sich nicht um unbewusste oder instinktive biologische Adaptionsprozesse handelt, sondern um bewusste individuelle Handlungsentscheidungen im professionellen Kontext.

Wajcmans Argumentation kommt zu einem ähnlichen Schluss, sie lehnt deterministische Deutungsmuster ebenso entschieden ab und schreibt:

„I have suggested that all technologies be properly characterized as contingent and open, expressing the networks of social relations in which they are embedded. With this in mind, we will be less inclined to identify technology itself as the source of positive or negative change, and will concentrate instead upon the changing social relationships within which technologies are embedded and how technologies may facilitate or constrain those relationships." (Wajcman, 2004, S. 108)

Weiterhin betont sie, dass technologische Innovationen vor allem deshalb neue Möglichkeitsräume schaffen, weil es Frauen gibt, die diese neuen Räume für sich einnehmen und die diesen Räumen zugrunde liegenden Maschinen nicht als männliche Domäne betrachten. Daraus ableitend bewertet sie die Förderprogramme, die junge Frauen vermehrt in technische Berufe bringen sollen, als positiven Schritt auf dem Weg zu mehr Gleichberechtigung (ebd., S. 109), wobei sie, differenztheoretisch argumentierend, bestehende Hürden vor allen Dingen in der Fortschreibung der Dominanz patriarchaler Strukturen sieht, die Frauen ihrer Ansicht nach davon abhalten, sich entsprechend zu professionalisieren (ebd., S. 112). Des Weiteren 
weist Wajcman auf das fehlende Problembewusstsein innerhalb der Praxis hin, wodurch sie mehr oder weniger direkt die Relevanz der hier fortgeführten Auseinandersetzung unterstreicht:

„Practitioners act as if their own methodologies are not affected by the social context and have no politics. They do not reflect on how the preponderance of white, privileged, heterosexual men might have framed the field. [...] For technofeminism, politics is an 'always-already' feature of a network, and a feminist politics is a necessary extension of network analysis. Science and technology embody values, and have the potential to embody different values.“" (ebd., S. 126)

Haraway bedient sich ebenfalls des Co-Emergenzprinzips, indem sie in ihrer Kernschrift feministischer Technikforschung, dem Cyborg Manifesto, herausarbeitet, wie Mythos und Werkzeug sich wechselseitig konstituieren. Technologien und (Geschlechter-)Diskurse sind für sie gleichermaßen geronnene Momente fluider sozialer Interaktionen (Haraway, 1985/2007, S. 45). Haraway versteht Geschlecht als Mythos, der sich durch Performanz und Formen des Doing Gender innerhalb sozialer Interaktion konstituiert und in Wechselwirkung zu technologischen Entwicklungen steht. Haraway sieht in der Figur der Cyborg und in ihrer Metaphorik als Chimäre zwischen Mensch und Maschine die Möglichkeit, klassisch dualistische und differenzialistische Denkmuster aufzubrechen, was in ihrer Interpretation feministische Ermächtigungsstrategien unterstützt. Diese gesellschaftswissenschaftlichen Implikationen greifen auch Flanagan et al. auf und formulieren für die gestalterische Praxis, deren Verantwortung im Hinblick auf ihre Rolle im Diskurs sie betonen, den klaren Anspruch, die in technologische Artefakte eingeschriebenen sozialen Werte als Kriterium der Beurteilung der Qualität von Technologie zu berücksichtigen.

„If an ideal world is one in which technologies promote not only instrumental values such as functional efficiency, safety, reliability, and ease of use, but also the substantive social, moral, and political values to which societies and their people subscribe, then those who design systems have a responsibility to take these latter values as well as the former into consideration as they work In technologically advanced, liberal democracies, this set of values may include liberty, justice, enlightenment, privacy, security, friendship, comfort, trust, autonomy and sustenance." (Flanagan et al., 2008, S. 322)

Im Hinblick auf die Differenzierungskategorie Geschlecht und ihre Fortschreibung im Bereich der IT ist das Bewusstsein um die hier angesprochene Verantwortung der gestalterischen Praxis zentral.

Im eingangs aufgegriffenen Anspruch, die „Frauenperspektive“ zwecks erfolgreicher Kommunikation zu berücksichtigen, spiegelt sich die bewusste Entscheidung, sich der Praxis des Doing Gender im Bereich des IT-Designs anzuschließen und die Mann-Frau-Dichotomie mit all ihren Implikationen und inhärenten Hierarchisierungen innerhalb der Gestaltungspraxis aufrecht zu erhalten - was nachfolgend, im Hinblick auf mögliche Auswirkungen dieser Praxis, anhand verschiedener Beispiele problematisiert wird. 


\section{Doing Gender im IT-Design: (Re-)Konstruktion oder Überwindung binärer Differenzkategorien}

Bereits ein kritischer Blick in die IT-Geschichte zeigt, dass die hier und jetzt formulierten Wünsche nach mehr Beteiligung von Frauen im IT-Bereich im Grunde Reclaims längst erreichter Zielen darstellen. Light (1999) hat hier im Bereich der Technikgeschichte mit ihrer Studie zur zentralen Rolle von Technikerinnen in der frühen Entwicklungsphase von elektronischen Computern im doppelten Sinne Pionierinnenarbeit geleistet. Ihre Auseinandersetzung mit den Ursprüngen der Computerentwicklung in den USA während des Zweiten Weltkriegs ergab, dass die maßgeblich an der Konstruktion des ENIAC (Electronic Numerical Integrator and Computer, erster rein elektronischer Universalrechner) beteiligten Technikerinnen gezielt aus der IT-Entwicklungsgeschichte herausgeschrieben wurden. Ihre Professionalität wurde ihnen aberkannt, ihre „Weiblichkeit“ hingegen wurde in den Vordergrund gerückt.

„While celebrating women's presence, wartime writing minimized the complexities of their actual work. While describing the difficulty of their task, it classified their occupations as subprofessional. While showcasing them in formerly male occupations, it celebrated their work for its femininity. Despite the complexities - and often pathbreaking aspects - of the work women performed, they rarely received credit for innovation or invention." (Light, 1999, S. 455)

Hier wurde die Tätigkeit im IT-Bereich klar vergeschlechtlicht bewertet: „,The ENIAC project made a fundamental distinction between hardware and software: designing hardware was a man's job; programming was a woman's job.“ (Light, 1999, S. 468). Kathleen McNulty oder Frances Bilas waren die ersten Programmiererinnen. Sie übten Tätigkeiten im Bereich der Softwareentwicklung aus, für die weitreichende Kenntnisse und Fähigkeiten im Umgang mit Hardware notwendig waren. Die konstruierte Dichotomie stieß auch damals unmittelbar an ihre Grenzen, wurde aber insoweit auf die Spitze getrieben, als entlang des Geschlechts überhaupt erst definiert wurde, welche Tätigkeiten als „,technologisch“ galten - wenn Frauen also IT-Berufe ausübten, waren es keine - prestigeträchtigen - IT-Berufe mehr (ebd, S. 468 ff.). Ihre beruflichen Erfolge wurden von Anfang an negiert, in den Ergebnispräsentationen, die die Frauen selbstverständlich vorbereitet hatten, und in den Dokumentationen der Entwicklungsleistungen kamen ihre Namen schlichtweg nicht mehr vor, genauso wenig im öffentlichen Diskurs um die technologischen Innovationen, die sie maßgeblich mit erarbeitet hatten - nicht einmal dann, wenn sie, wie Adele Goldstine, in Führungspositionen aufgestiegen waren und sich breite technologische Expertise erarbeitet hatten (ebd., S. 472 ff.). Erschreckend deutlich wird die Hierarchisierung innerhalb der Mann-Frau-Differenz in diesem Zusammenhang auch in der zugehörigen Management-Literatur der 1940er Jahre. Light zitiert aus einem Leitfaden für Manager: „Women can be trained to do any job you've got but remember 'a woman is not a man'; A woman is a substitute - like plastic instead of metal.“ (Light 1999, S. 481). Deutlicher lassen sich die Verhältnisse innerhalb der Zuschreibungstrias Gender, Technologie und Arbeit nicht aufzeigen.

Mit ihrer historischen Analyse hat Light einen wichtigen Beitrag zur Dekonstruktion des Mythos, dass Frauen desinteressiert an IT und/oder unfähig seien, geleistet. Das ENIACBeispiel ist exemplarisch für einen generalisierbaren Prozess. Klaus formuliert aus der Perspektive der Gender Media Studies: 
„Die Frau ist keine Essenz, sondern eine Form des Mythos ,Geschlecht', die mit gesellschaftlichen, kulturellen und historischen Bedeutungen gefüllt ist. Durch die geschlechtliche Identifikation der Individuen wird der Mythos zur Basis ihrer Weltaneignung. Die Bedeutung und die Anreicherung des Mythos mit Sinn geschieht nicht von außen, wird den Menschen nicht oktroyiert, sondern von den sozialen Subjekten im Lebensvollzug, in ihrem kontextgebundenen Handeln erschaffen.“ (Klaus, 2005, S. 361)

Sie betont dabei nachdrücklich, dass die Kategorie Geschlecht keineswegs das Handeln determiniere und gibt, ähnlich wie Light, Beispiele aus der Berufsfeldforschung an. Diese beschreibt den Wandel zahlreicher Berufe vom typischen Männer- zum ebenso typischen Frauenberuf und umgekehrt (ebd.). Problematisch und im wörtlichen Sinne bezeichnend ist dabei, dass mit diesem Wandel zugleich ein Wandel von materieller und immaterieller Wertigkeit des Berufs verbunden ist, dass bei gleicher Tätigkeit deutliche Differenzen im Bereich Einkommen und Bezahlung sowie in Fragen der allgemeinen Anerkennung und Prestigeträchtigkeit reproduziert werden. Befindet sich ein Beruf in einer Phase „Männerberuf“ zu sein, gilt die selbe professionelle Tätigkeit als respektabler und prestigeträchtiger und wird materiell höher entlohnt, als in der Phase, in der er als „Frauenberuf“" kategorisiert wird. In extremen Fällen geht die Entwicklung so weit, dass der weiblich konnotierten Tätigkeit der Berufsstatus selbst aberkannt wird, was man beispielsweise in den historischen Entwicklungen im Bereich der Pflege- und Erziehungstätigkeiten beobachten kann.

Gildemeister und Wetterer beschreiben einen geschlechterdualistisch segregierten Arbeitsmarkt und bestätigen die historische Analyse von Light in Bezug auf Frauen in IT-Berufen. Neben dem Wandel von der Programmiererin zum Programmierer und der entsprechenden Aufwertung des Berufs beobachten sie ähnliche Prozesse des „Geschlechtswechsels“ von beruflicher Tätigkeit auch für andere Berufsfelder. Markante Beispiele sind der Wechsel vom Sekretär zur Sekretärin, einhergehend mit einer entsprechenden Abwertung, wobei sprachliche Atavismen mit altem Status erhalten bleiben (Parteisekretär, UN-Generalsekretär), der Wechsel von der Hausfrau zum Koch/Gebäudereiniger, mit der entsprechenden Aufwertung und Anerkennung der Tätigkeit als „Arbeit“. Diese diskursive Strategie lässt sich auch heute noch beobachten - besonders auffällig im Bereich der Kindererziehung bzw. den Wechsel von der Mutter zum Erzieher betreffend. Auch wenn die Anerkennung erzieherischer Tätigkeit als Arbeit bereits eine längere Tradition hat, fängt die Debatte um ihre Wertigkeit, sprich um höhere Löhne und mehr Prestige, erst an, als vermehrt auch Männer den Beruf ergreifen. (Diese Facette des Aushandlungsprozesses zeigt sich beispielsweise in der Berichterstattung um den von ver.di initiierten Kita-Streik im Jahr 2012. ${ }^{3}$ Die hier unkritisch formulierte Darstellung des Gender Pay Gap - „Netto hatten Erzieherinnen nach Zahlen des Mikrozensus 2008 im Schnitt 1365 Euro zur Verfügung, die wenigen Männer rund 230 Euro mehr.“ - lässt sich zusätzlich zum angesprochenen Aspekt in die Gesamtproblematik der vergeschlechtlichten Wertung von Arbeit einordnen.) Des Weiteren stellen sie - auch historisch betrachtet den Wandel von der Heilkundigen Frau zum Arzt dar, der mit einer massiven Aufwertung der Tätigkeit einherging, sowie die Aufwertung der Arbeit der Schriftsetzerin durch den Setzer und die damit einhergehende Gleichsetzung von Männlichkeit und Maschinenarbeit. Ihr

\footnotetext{
${ }^{3}$ Vgl dazu bspw.: http://www.focus.de/finanzen/news/arbeitsmarkt/tid-25265/miese-bezahlung-kaum-anerkennungwarum-die-kita-mitarbeiter-auf-die-strasse-gehen-was-erzieher-verdienen_aid_723939.html (letztmaliger Zugriff: 11.05.2014).
} 
Fazit fällt dabei deutlich aus: Inhalte, die Geschlechterdifferenz ausmachen, sind beliebig und ändern sich im Laufe der Geschichte, Geschlecht gilt als Statuskategorie und funktioniert als Platzanweiser oder „Allokationsmechanismus“, Gleichheit wird oftmals als Tabu betrachtet und im Bereich des Arbeitsmarktes gibt es keine „,androgynen Berufe“, sondern im Zweifelsfall „rosa und hellblau gefärbte Teilbereiche“. Hierarchie und Differenz wird also immer wieder neu hergestellt (Gildemeister und Wetterer, 1992, S. 226 ff.). Auch 20 Jahre nach dieser Analyse sind der Arbeitsmarkt und die Bewertung von Leistungen noch stark in geschlechtsgebundene Segmente unterteilt, allerdings gibt es ebenso Berufs- und Tätigkeitsfelder, die sich von der Vergeschlechtlichung professioneller Handlungsfelder abwenden und/oder für gerechtere Ressourcenverteilung sorgen, was für den IT-Bereich, wie für den Bereich öffentlicher Kommunikation insgesamt, eine große Chance darstellt.

An den oben genannten Beispielen werden Kernerkenntnis und Kerndilemma der Geschlechterforschung gleichsam deutlich - einerseits die Erkenntnis, dass Geschlecht nicht handlungsdeterminierend ist, bzw. dass Zweigeschlechtlichkeit sich als soziales Konstrukt über Interaktion und Institutionalisierung etabliert hat und damit keine grundsätzlich gegebene Konstante darstellt, andererseits die Hierarchisierung innerhalb der als dualistisch konstruierten Geschlechterordnung, die für Ungerechtigkeiten und wiederkehrende Abhängigkeitsverhältnisse sorgt. Wird ein Berufsfeld „,weiblich“, wird es zugleich - oft bis zur materiellen Wertlosigkeit bzw. Aberkennung des „Arbeits-“Status - entwertet, wird ein Berufsfeld „männlich“, findet eine materielle Aufwertung statt, mit der eine höhere gesellschaftliche Anerkennung der Tätigkeit einher geht. Vielfältigere Geschlechteridentitäten spielen innerhalb dieses Prozesses keine Rolle, werden nicht anerkannt. Diesen sich selbst wiederholenden Prozess gilt es aufzubrechen - auch und gerade durch Irritation der vergeschlechtlichten Wahrnehmung menschlicher Kommunikation in der IT.

Speziell für den Bereich der IT wird deutlich, wie auf der Konstruktions- und Design-Ebene im Entstehungsprozess normativ gebundene Entscheidungen getroffen werden, die Wertungen vorgeben und Handlungsspielräume systematisch einschränken können (Jelden, 1999). Dieser Perspektive wird, neben anderen Teilaspekten der Konstruktion und Dekonstruktion von Geschlecht, auch im Band von Zorn (2007) nachgegangen. Während also das Ziel, mehr Frauen in IT-Berufe zu bringen und damit für einen Ausgleich innerhalb der Branche und für gendersensibles Design von Kommunikationsprodukten zu sorgen, aus gerechtigkeitspolitischer Perspektive heraus sinnvoll und gut erscheint, muss man sich gerade in den gestalterischen Berufen im Feld der Informations- und Kommunikationstechnologie - darüber bewusst werden und kritisch reflektieren, inwieweit man selbst zu den hier dargestellten Praktiken des Doing Gender und den damit verbundenen Hierarchisierungspraktiken (un-)intendiert beiträgt. Um mit Gildemeister und Wetterer zu sprechen: Die ständige Wiederholung und damit Festigung eines dualistischen Menschenbildes und seine Einschreibung in Technologien der digitalen Kommunikation erweisen sich als problematisch - vor allem im Bewusstsein um die Konstruiertheit der binären Geschlechterordnung und im Verständnis von Geschlechtsidentität als fortlaufendem Herstellungsprozess.

Der hier diskutierte handlungstheoretische Rahmen liefert eher das notwendige emanzipatorische Potential als die klassischen Differenz- und Determinismustheorien. Er soll der gestalterischen Praxis an dieser Stelle eines mitgeben: den Mut zu Irritation und zu Brüchen mit den bestehenden Gender-Dualismen und rosa-blau „Farbschemata“ und ihren inhärenten 
sozialen Implikationen und Hierarchisierungen - und das ohne sich selbst vom Gleichheitstabu einengen $\mathrm{zu}$ lassen.

\section{Literatur}

Becher, V. Bönninghausen, I., von Remus, U., Schwarz, K. Wilhelm, U. \& Zimmermann, R. (1981). Die Situation der Mitarbeiterinnen im WDR, Köln.

Butler, J. (2002). Performative Akte und Geschlechterkonstitution - Phänomenologie und feministische Theorie. In U. Wirth (Hrsg.), Performanz - Zwischen Sprachphilosophie und Kulturwissenschaften (S. 301-322.). Frankfurt a. M.: Suhrkamp.

Dogruel, L. (2013). Eine kommunikationswissenschaftliche Konzeption von Medieninnovationen. Begriffsverständnis und theoretische Zugänge. Wiesbaden: Verlag für Sozialwissenschaften.

Flanagan, M., Howe, D \& Nissenbaum, H. (2008). Embodying Values in Technology - Theory and Practice. In: J. van den Hoven \& J. Weckert (Hrsg.), Information Technology and Moral Philosophy (S. 322-353). Cambridge: Cambridge University Press.

Gildemeister, R. (2008). Soziale Konstruktion von Geschlecht: „Doing Gender“. In S. Wilz (Hrsg.), Geschlechterdifferenzen - Geschlechterdifferenzierungen: Ein Überblick über gesellschaftliche Entwicklungen und theoretische Positionen (S. 167-198). Wiesbaden: Verlag für Sozialwissenschaften.

Gildemeister, R. \& Wetterer, A. (1992). Wie Geschlechter gemacht werden. Die Soziale Konstruktion der Zweigeschlechtlichkeit und ihre Reifizierung in der Frauenforschung. In G.-A. Knapp \& A. Wetterer (Hrsg.), TraditionenBrüche. Eintwicklungen feministischer Theorie (S. 201-254). Freiburg i.Br.: Kore Verlag.

Haraway, D. (1985). A Cyborg Manifesto: Science, Technology and Socialist-Feminism in the late 20th century. In D. Bell \& B. Kennedy (Hrsg.), The cybercultures reader (S. 34-65). London/New York: Routledge.

Jelden, E. (1999). Frauen am Computer: Männlich programmiert? In M. Ritter (Hrsg.), Bits und Bytes vom Apfel der Erkenntnis. Frauen - Technik - Männer (S. 156-170). Münster: Westfälisches Dampfboot

Klaus, E. (2005). Kommunikationswissenschaftliche Geschlechterforschung. Zur Bedeutung der Frauen in den Massenmedien und im Journalismus. Berlin/Münster: LIT Verlag

Klaus, E. (2001). Ein Zimmer mit Ausblick? Perpektiven kommunikationswissenschaftlicher Geschlechterforschung. In J. Röser \& U. Wischermann (Hrsg.), Kommunikationswissenschaft und Gender Studies (S. 20-40). Wiesbaden: Verlag für Sozialwissenschaften

Light, J. (1999). When Computers Were Women. Technology and Culture, 40(3), 455-483.

Lünenborg, M. \& Maier, T. (2013). Gender Media Studies, Konstanz: UTB

Lünenborg, M., Röser, J., Maier, T., Müller, K. \& Grittmann, E. (1999). Merkels Dekolleté als Mediendiskurs. Eine Bild-, Text- und Rezeptionsanalyse zur Vergeschlechtlichung einer Kanzlerin. In M. Lünenborg (Hrsg.), Politik auf dem Boulevard? Die Neuordnung der Geschlechter in der Politik der Mediengesellschaft (S. 73-102). Bielefeld: transcript

Neverla, I.(1983). Arbeitsmarktsegmentation im journalistischen Beruf. Publizistik, 3, 343-362.

Neverla, I. \& Kanzleiter, G. (1984). Journalistinnen. Frauen in einem Männerberuf, Frankfurt a. M.: Campus

Röser, J. \& Müller, K. (2012). Merkel als „einsame Spitze“ - Eine quantitative Inhaltsanalyse zum Geschlechterverhältnis von Spitzenkräften in den Medien. In M. Lünenborg \& J. Röser (Hrsg.), Ungleich mächtig. Das Gendering von Führungspersonen aus Politik, Wirtschaft und Wissenschaft in der Medienkommunikation (S. 37-64). Bielefeld: transcript 
Scheich, E. (1999). Technologische Objektivität und technische Vergesellschaftung - Identitätslogik im naturwissenschaftlichen Diskurs. Zur Veränderung erkenntnistheoretischer Perspektiven durch die Konstruktion und Politisierung der Natur. In M. Ritter (Hrsg.), Bits und Bytes vom Apfel der Erkenntnis. Frauen - Technik - Männer (S. 76-104) Münster: Dampfboot Verlag

Tuchman, G. (1978). The Symbolic Annihilation of Women by the Mass Media. In G. Tuchman (Hrsg.), Hearth and Home. Images of Women in the Mass Media (S. 3-38) New York: The University of Chicago Press

Wajcman, J. (2004). TechnoFeminism, Cambridge: Polity Press

Winner, L. (1986). The Whale and the Reactor. A Search for Limits in an Age of High Technology. Chicago/London: University of Chicago Press

Zorn, E., Maass, S., Rommes, E., Schirmer, C. \& Schelhowe, H (Hrsg.) (2007). Gender Designs ITConstruction and Deconstruction of Information Society Technology. Wiesbaden: Verlag für Sozialwissenschaften 